Nechani cal properti es and rei nf or cement of $\mathrm{Bi}-2212$ t ubul ar bul $\mathrm{k}$ super conduct or for current I ead

\begin{tabular}{|l|l|}
\hline $\begin{array}{l}\text { jour nal or } \\
\text { publ i cat i on } \mathrm{titl} \text { e }\end{array}$ & I EEE Transact i ons on Appl i ed Super conduct i vi ty \\
\hline vol une & Vol . 12 \\
\hline number & I ssue 1 \\
\hline page $\mathrm{r}$ ange & pp. 1319-1322 \\
\hline year & 2002-03 01 \\
\hline URL & ht t p: //hdl . handl e. net /10655/2238 \\
\hline
\end{tabular}




\title{
Mechanical Properties and Reinforcement of Bi-2212 Tubular Bulk Superconductor for Current Lead
}

\author{
Hitoshi Tamura, Toshiyuki Mito, Akifumi Iwamoto, Yutaka Yamada, and Kyoji Tachikawa
}

\begin{abstract}
High-Tc superconductor has been expected as a current lead between a low Tc superconductor and a power supply for a large-scale superconducting magnet system because of its advantages of a heat load to a cryogenic system and a high transfer current density. Bi-2212 bulk superconductor is a candidate material for this kind of current lead. To design a current lead using High-Tc superconductor, we investigated mechanical properties of a Bi-2212 bulk which was prepared by a diffusion process. A concept design of a current lead reinforced by using a glass-epoxy was considered.
\end{abstract}

Index Terms-Bi-2212, current lead, high temperature superconductor, mechanical reinforcement.

\section{INTRODUCTION}

$\mathbf{T}$ HE PERFORMANCE of high-Tc superconductor (HTS) such as $\mathrm{Bi}-\mathrm{Sr}-\mathrm{Ca}-\mathrm{Cu}-\mathrm{O}$ system or $\mathrm{Y}-\mathrm{Ba}-\mathrm{Cu}-\mathrm{O}$ system has improved rapidly. They have been expected as a current feeder between a low-Tc superconductor and a current transport material at higher temperature. Each HTS has its own characteristic; the $\mathrm{Bi}-2212$ system has a high transfer current density at cryogenic temperature, the critical temperature of $\mathrm{Bi}-2223$ is much higher than liquid nitrogen temperature, and the YBCO system is useful under high magnetic field conditions. They also have the advantage of low heat load to a cryogenic system. By using HTS for a current lead, which is connected between the power supply and the superconducting bus line, the heat load could be reduced to about $1 / 4$ of a conventional helium gas cooled copper current lead [1].

Yamada et al. has developed Bi-2212 bulk material by using diffusion process [2], [3]. Current transport tests for the tubular bulk at $4 \mathrm{~K}$ have been conducted at NIFS under a research collaboration program. The maximum transport current density of $35 \mathrm{kA} / \mathrm{cm}^{2}$ was successfully achieved at the diffusion layer so far. However, in these current transport tests, some specimens were broken or got a structural damage, which were caused by the thermal stress in the bulk specimen when a part of a surface of the specimen transferred to normal conductivity. HTS naturally has a disadvantage concerning with mechanical strength. It is needed to reinforce the bulk superconductor or to fix the bulk to an appropriate support structure as a size becomes large, especially for a practical use. Since HTS is made of oxide, it is a brittle material and its thermal contraction would be small.

Manuscript received September 24,2001. This work was supported in part by Grant-in-Aid from Ministry of Education, Culture, Sports, Science and Technology of Japan under Grant 12750086.

H. Tamura, T. Mito, and A. Iwamoto are with the National Institute for Fusion Science, Toki, Gifu 509-5292, Japan (e-mail: tamura@nifs.ac.jp).

Y. Yamada and K. Tachikawa are with Tokai University, Hiratsuka, Kanagawa 259-12, Japan (e-mail: yyamaday@keyaki.cc-tokai.ac.jp).

Publisher Item Identifier S 1051-8223(02)04188-X.

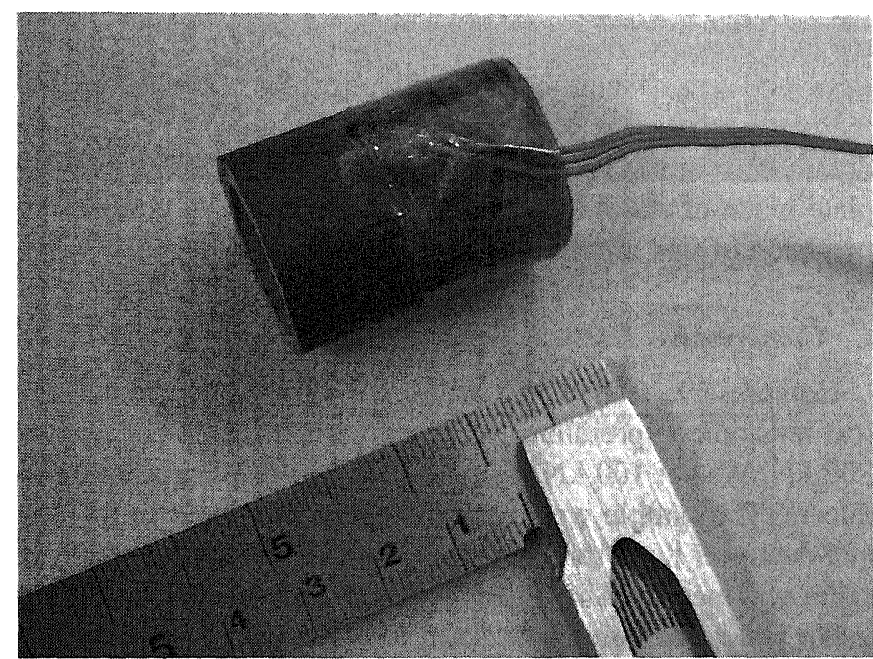

Fig. 1. A specimen for thermal contraction and compressive test.

Rigidity reinforcement and absorption of thermal contraction during cooling down have to be considered simultaneously for a design of a current lead using HTS.

To know the mechanical properties of HTS bulk material such as thermal expansion coefficient, Youngs modulus and tensile/compressive strength is very important for developing an optimum reinforce method. We measured mechanical properties of $\mathrm{Bi}-2212$ tubular bulk materials prepared by diffusion process and investigated an optimal design of HTS current lead using this material.

\section{EXPERIMENTS}

\section{A. Specimen}

The original size of the $\mathrm{Bi}-2212$ superconductor used for mechanical tests was $27 \mathrm{~mm} / 19 \mathrm{~mm}$ in outer/inner diameters and $200 \mathrm{~mm}$ of length, respectively. This was the same size and structure with the specimen for current transfer experiments. The specimen for the mechanical tests were obtained from this tubular bulk. A nominal length of the specimen was $30 \mathrm{~mm}$. Since the Bi-2212 is a $0.15 \mathrm{~mm}$ thick layer on both outer and inner surfaces and a residual region is a bismuth-free substrate, the tubular bulk is not an isotropic material. To compare the mechanical characteristics between Bi-2122 bulk superconductor and a substrate, which is an isotropic, the substrate specimen was also prepared. The composition ratio of the substrate is $\mathrm{Bi}: \mathrm{Sr}: \mathrm{Ca}: \mathrm{Cu}=0: 2: 1: 2$. The $\mathrm{Bi}-2212$ layer was synthesized on this substrate by coating and reacting $\mathrm{Bi}-\mathrm{Cu}$ oxide with $\mathrm{Ag}_{2} \mathrm{O}$. A silver layer was generated on the outer surface of $\mathrm{Bi}-2212$ [4]. 


\section{B. Thermal Contraction}

The thermal contraction between room temperature and $77 \mathrm{~K}$ were measured for both the Bi-2212 and the bismuth-free substrate. Strain gauges were attached on the surface of the specimen. Thin copper and 304 stainless steel plates, on which the same lots of gauges were attached, were used for reference. The thermal contraction of the object was calculated by subtracting the theoretical thermal contraction of the reference plate from the measured strain value of the specimen, and then adding the measured strain value of the reference plate. The theoretical thermal contraction of the material of the reference plate was obtained from the Cryocomp package of Cryodata Inc. [5]. A plate made of stainless steel 304 was prepared to confirm the precision of this measurement.

\section{Compressive Test}

Compressive tests were carried out with an electromechanical tension/compression test system which has a capacity of $200 \mathrm{kN}$. We set $100 \mathrm{kN}$ load cell and two displacement transducers. The sample was placed between the upper fixed plate and the lower thick stainless steel plate. A height change of this space was measured by using the displacement transducers. The lower plate was on a stage fixed to a moving cross head. The speed of the moving cross head was set to $0.2 \mathrm{~mm} / \mathrm{min}$. The tests were carried out at room temperature except for bismuth-free substrate. The substrate was cooled in liquid nitrogen and tested at atmosphere keeping its temperature by supplying the coolant around the specimen. In this case, the displacement transducers could not be mounted so that a displacement of the moving cross head was recorded.

\section{RESULTS}

\section{A. Thermal Contraction}

Apparent strains of the copper plate and stainless steel plate from $300 \mathrm{~K}$ to $77 \mathrm{~K}$ were $-127 \mu \varepsilon$ and $145 \mu \varepsilon$, respectively. Since the theoretical thermal contraction of copper is $0.3142 \%$, the calculated thermal contraction of the stainless steel would be $0.2870 \%$ while the theoretical value is $0.2967 \%$. The accuracy of this measurement was estimated to be $0.01 \%$. The thermal contraction from $300 \mathrm{~K}$ to $77 \mathrm{~K}$ of the $\mathrm{Bi}-2212$ bulk was $0.20 \%$ that was as well as that of bismuth free substrate. There was no significant difference between them concerning overall thermal contraction.

\section{B. Compressive Strength and Young's Modulus}

Stress and strain were both calculated from the measured compressive load and the displacement with an actual size of the cross sectional area and the length of the specimen. Fig. 2 and 3 show the stress-strain curves of the Bi-2212 bulk and the bismuth-free substrate at room temperature, respectively. The compression was loaded until the specimen broke. All specimens were broken without plastic deformation as shown in Fig. 4. Fig. 5 shows stress against cross head displacement of the substrate at $77 \mathrm{~K}$. It seemed that the compressive strength at $77 \mathrm{~K}$ did not change so much compared to the result at room temperature.

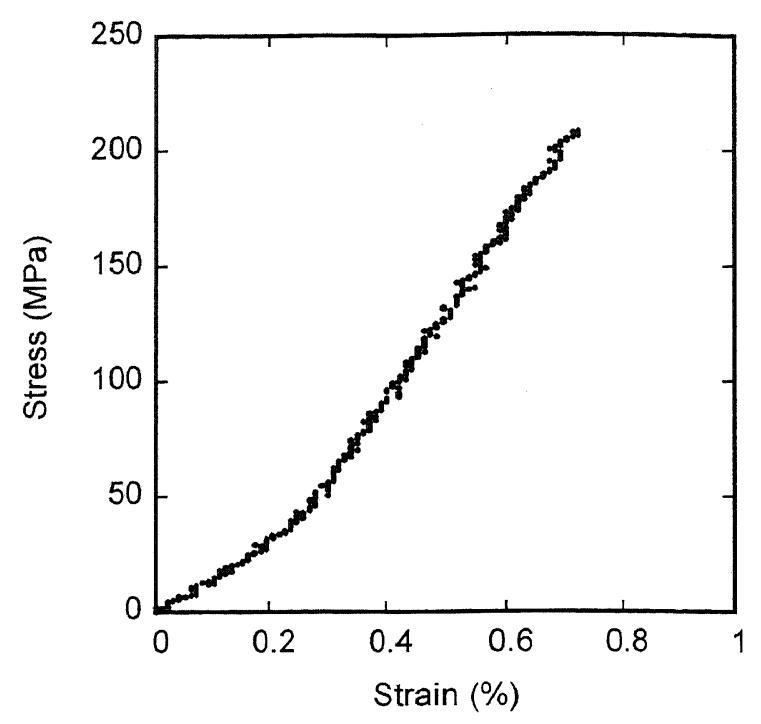

Fig. 2. Stress-strain curve of Bi-2212 tubular bulk at room temperature.

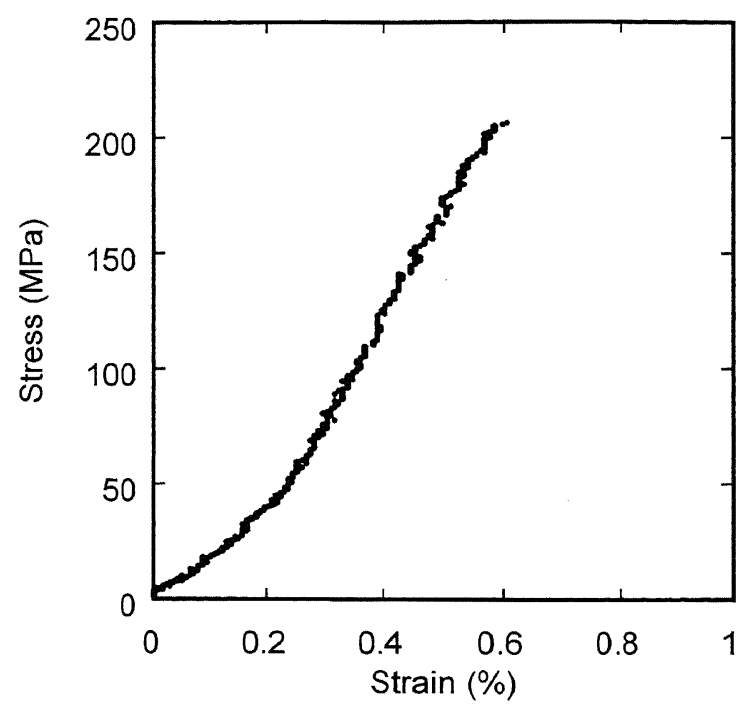

Fig. 3. Stress-strain curve of Bi-free substrate at room temperature.

We estimated the Young's moduli of the materials from linear behavior region in a stress-strain curves; omitting an initial load condition which showed low rigidity since the specimen did not touch completely to the tester. The estimated Young's modulus of $\mathrm{Bi}-2212$ bulk tube and bismuth-free substrate were $37.4 \mathrm{GPa}$ and $44.5 \mathrm{GPa}$, respectively.

\section{DISCUSSIONS}

The diffusion layer of the Bi-2212 HTS consists of thin-plate like grains grown to the radial direction [4]. This could be the reason why the thermal contraction shows no difference between HTS and its substrate. There is also a silver layer on the diffusion layer, but the silver layer must have reached yield stress caused by the difference of the thermal contraction against the substrate. Consequently, the thermal contraction of the HTS yields to that of the substrate. 


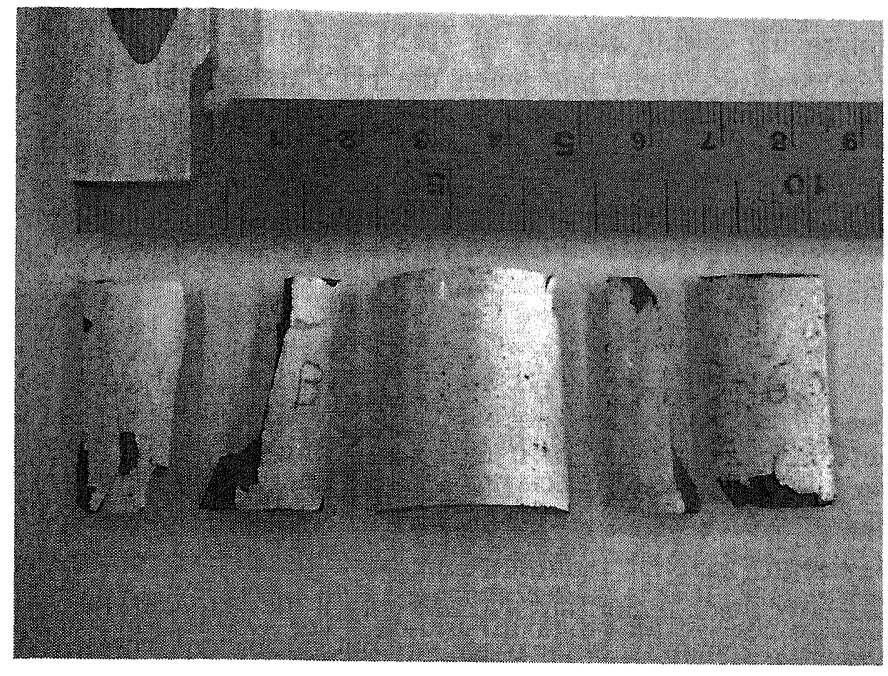

Fig. 4. Photograph of fracture mode.

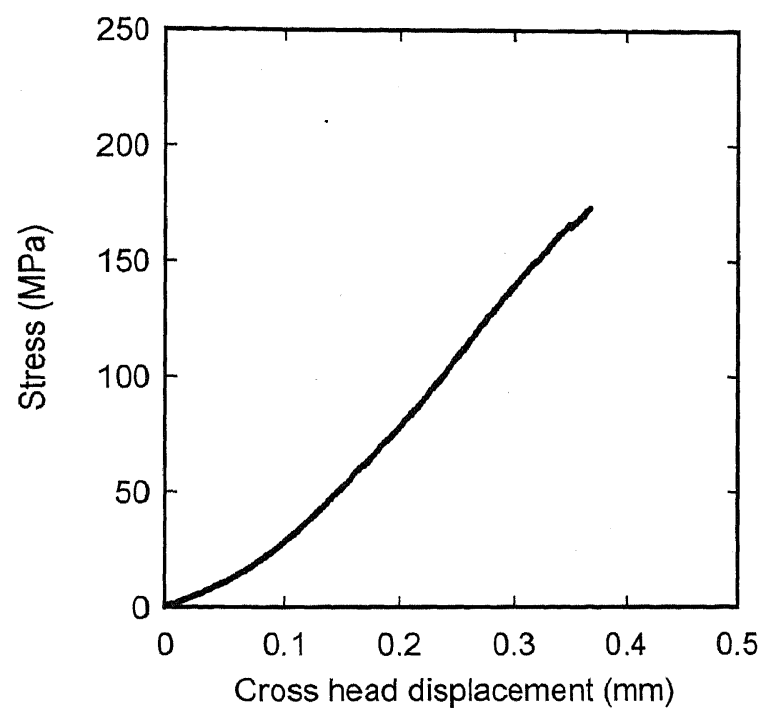

Fig. 5. Stress against cross head displacement of Bi-free substrate at $77 \mathrm{~K}$.

The compressive strength among the HTS, the substrate (both at $300 \mathrm{~K}$ and $77 \mathrm{~K}$ ) were not so different. The substrate of the HTS must be a main member against load. Once an initial crack is generated in the substrate, the crack grows rapidly then reaches to fracture. It is needed to prevent a generation of a crack and to increase a resistance against crack growth or crack opening. The Bi-2212 diffusion layer may act as initial crack from its structure. Yamada et al. showed that three point bending strength of Stycast 2850 FT coated Bi-2212 bulk improved both at $77 \mathrm{~K}$ and $300 \mathrm{~K}$ [2]. Todate et al. reported some advantages of Stycast reinforced Bi-2223 HTS [6].

We made a prototype of fiber reinforcement Bi-2212 HTS by binding unidirectional glass/epoxy tapes to confirm a possibility of this reinforcement. The nominal thickness of the tape is $0.33 \mathrm{~mm}$ and volume fraction of epoxy is 24 to $30 \%$. The $19 \mathrm{~mm}$ in width tape was wrapped on the outer surface of the tubular HTS in two layers. A cure temperature and time of the glass/epoxy tape were $180^{\circ} \mathrm{C}$ and 15 hours, respectively. This glass/epoxy tape has high heat resistance so that it does not get

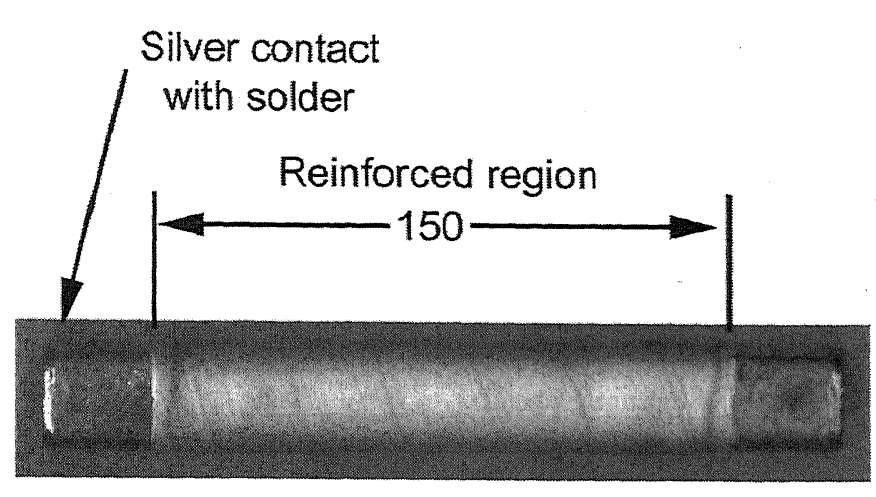

Fig. 6. Bi-2212 tubular bulk with glass-epoxy reinforcement.

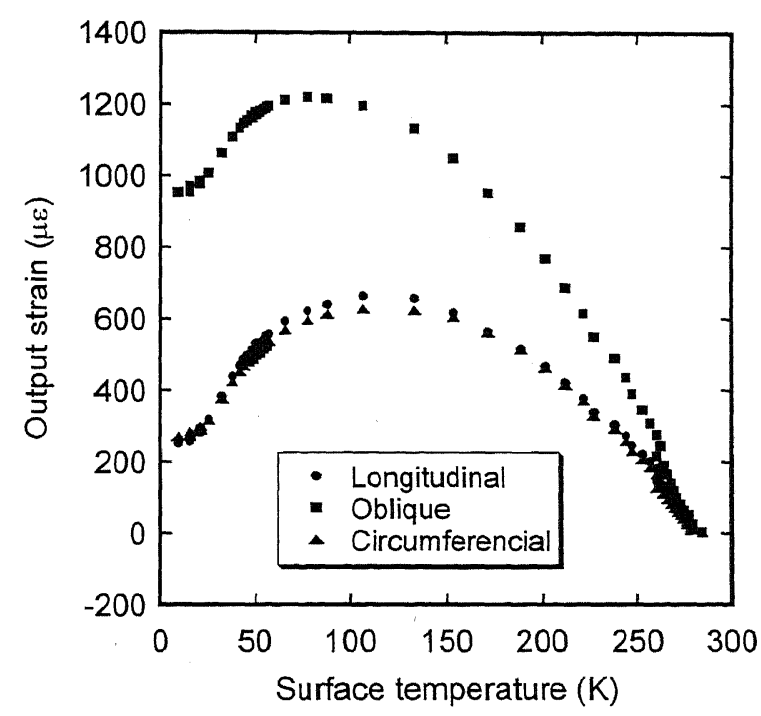

Fig. 7. Apparent strain against temperature obtained from strain gauges on the reinforced $\mathrm{Bi}-2212$ specimen.

damages during soldering contacts to terminals. Fig. 6 shows the manufactured prototype $\mathrm{Bi}-2212$ tubular bulk with glass-epoxy reinforcement.

We measured the thermal contraction between room temperature and $9 \mathrm{~K}$ of the reinforced $\mathrm{Bi}-2212$ by attaching strain gauges on it. Fig. 7 shows the apparent strain against temperature obtained from the specimen. An estimation of the thermal contraction was done according to the same calculation manner as mentioned in Section II-B, except the apparent strain which was compared with a measured data [7]. Although the strain gauges used in each measurement were not quite the same, a material composition and a heat treatment among the gauges were similar. The accuracy of estimated value at $9 \mathrm{~K}$ is less than $0.02 \%$. The estimation of the thermal contraction is shown in Table I. Only the oblique direction which almost coincides with the direction of glass fiber was smaller than that of others. This reinforcement is effective against a mechanical load. Furthermore, the reinforcement did not affect the electrical specification of the HTS and there was no generation of defect after several normal transitions in the current transport test. 
TABLE I

THERMal CONTRACTION OF Bi-2212 PREPARED BY DIFFUSION PROCESS

\begin{tabular}{lrr}
\hline \hline & \multicolumn{2}{c}{ Thermal contractin (\%) } \\
& $300 \mathrm{~K}$ to $77 \mathrm{~K}$ & $300 \mathrm{~K}$ to $9 \mathrm{~K}$ \\
\hline Bismuth-free substrate & 0.20 & - \\
Bi-2212 bulk & 0.20 & - \\
Bi-2212 with glass-epoxy tape & & \\
$\quad$ longitudinal & 0.20 & 0.24 \\
$\quad$ oblique & 0.15 & 0.17 \\
$\quad$ circumferencial & 0.20 & 0.23 \\
\hline \hline
\end{tabular}

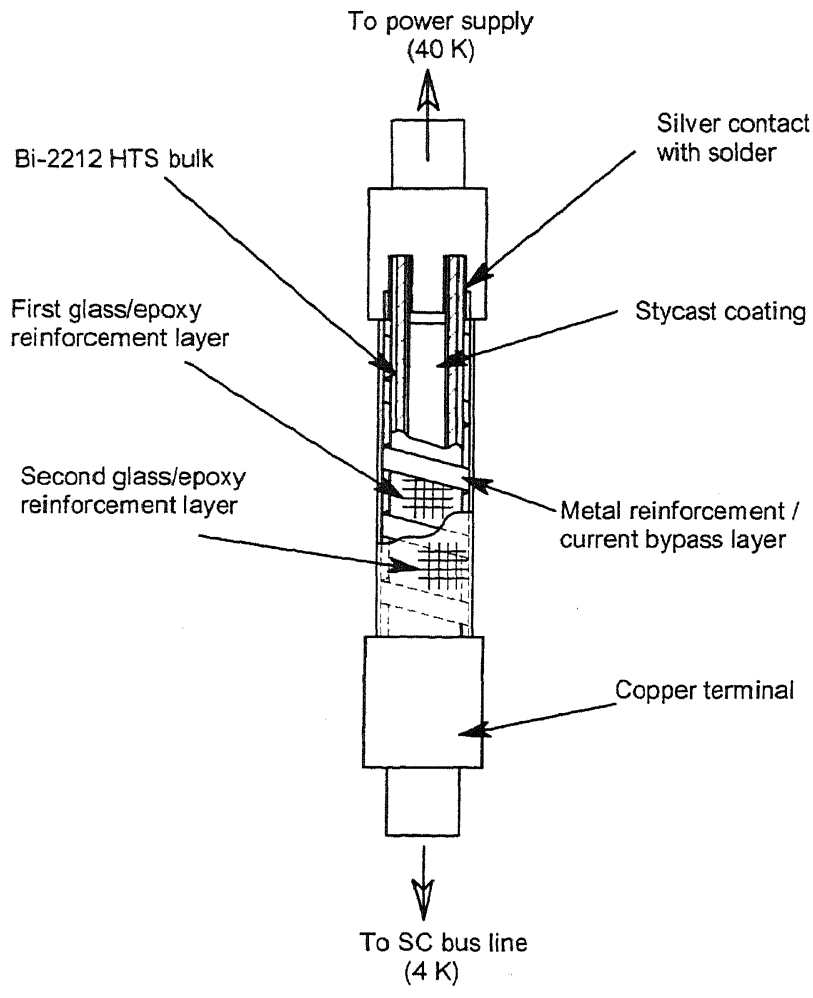

Fig. 8. Example design of current lead using Bi-2212 tubular HTS.

\section{CONCEPT DESIGN OF CURRENT LEAD}

The requirements for a current lead are; high transport current capacity, small heat leak to $4.2 \mathrm{~K}$ end, and stable under wide range of temperature. From this point of view, $\mathrm{Bi}-2212$ HTS prepared by diffusion process is regarded as the most likely candidate for current lead. To overcome breakage property, reinforcement by using high strength and low thermal conductive material is proposed.

Fig. 8 shows an example of current lead design using $\mathrm{Bi}-2212 \mathrm{HTS}$. The first reinforcement layer plays a role of improving overall rigidity of HTS. A spring shaped metal is a bypass current line when the HTS transfers to normal conductivity. It also acts as a deformation absorber against longitudinal tension/compression so that the material of this layer would be stainless steel. The second layer is to minimize a deformation caused by a misalignment, tensile, bend, torsion and compression.

\section{SUMMARY}

To study mechanical characteristics of $\mathrm{Bi}-2212 \mathrm{HTS}$ prepared by diffusion process for current lead, some mechanical properties of the HTS bulk material were investigated. Since the bulk is a brittle material, reinforcement using glass/epoxy would be effective since it can improve not only a mechanical strength but also a durability of the oxide without significant increase of thermal conductivity.

Attaching a spring shaped external support made of normal conducting material with high rigidity such as stainless steel is proposed for a current lead using this HTS bulk in points of a mechanical and an electrical stability.

\section{ACKNOWLEDGMENT}

The authors wish to express their thanks to Dr. T. Nagasaka of NIFS for his help in preparing specimens for mechanical test. They would like to thank also O. Suzuki and M. Enomoto of Tokai University for their cooperation on data acquisition during current transport tests.

\section{REFERENCES}

[1] T. Mito, K. Takahata, R. Heller, A. Iwamoto, R. Maekawa, and H. Tamura, et al., "Development of high temperature superconducting current feeders for a large-scale superconducting experimental fusion system," IEEE Trans. Appl. Superconduct., vol. 11, pp. 2611-2614, 2001.

[2] Y. Yamada, F. Yamashita, K. Wada, and K. Tachikawa, "Structure and superconducting properties of Bi-2212 cylinders prepared by diffusion process," Advances in Cryogenic Engineering (Materials), vol. 44, pp. 547-554, 1998.

[3] Y. Yamada, M. Takiguchi, O. Suzuki, K. Tachikawa, A. Iwamoto, H. Tamura, and T. Mito, "Transport performance of Bi-2212 current leads prepared by a diffusion process," IEEE Trans. Appl. Superconduct., vol. 10, pp. 1481-1484, 2000.

[4] Y. Yamada, O. Suzuki, M. Enomoto, K. Tachikawa, H. Tamura, A. Iwamoto, and T. Mito, "Performance of Bi-2212 HTS current leads prepared by the diffusion process," IEEE Trans. Appl. Superconduct., submitted for publication.

[5] "Cryocomp software," Eckels Engineering and Cryodata Inc., 1997.

[6] T. Todate, M. Matsukawa, K. Noto, Y. Yamada, K. Jikihara, and M. Ishihara, "Thermal conductivity of STYCAST reinforced Bi-2223 superconducting materials" (in Japanese), Cryogenic Engineering, vol. 30, pp. 495-501, 1995.

[7] A. Nishimura, H. Tamura, S. Imagawa, T. Mito, and Y. Yamaura et al., "Thermal and mechanical strain measurement of support structure of the large helical device," in Proc. ICEC 17, Bournemouth, UK, 1998, pp. $847-850$. 